\title{
6G Vision: Towards Future Collaborative Cognitive Communication (3C) Systems
}

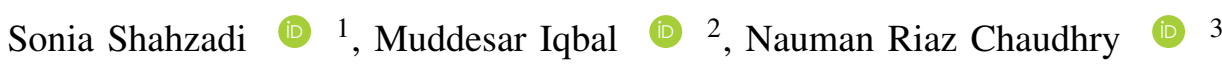 \\ ${ }^{2}$ Cognitive Systems Research Centre, School of Engineering, London South Bank University, London, UK \\ ${ }^{1,3}$ Department of Computer Science, University of Gujrat, Gujrat, Pakistan
}

\begin{abstract}
6G networks are expected to have a breakthrough by enabling the emergence of collaborative cognitive communication services over heterogeneous environments for industry 5.0 applications. These applications are required to adapt human-centric approach to make the most of human intuition and intelligence in Industry 4.0 automation.It calls for a transdisciplinarity research domain to investigate innovative systems with overlapping realms of Psychology, Sociology, Communication networks, Artificial Intelligence , Natural Language Processing and Collaborative Computing. The author at the Cognitive Systems Research Centre, London South Bank University has coined the expression "3C Systems" to refer to such artifacts which stands for "Collaborative Cognitive Communication Systems". In this paper, an innovative framework for 3C Systems is proposed that is able to analyze and predict both the human as well as machine behaviors. It proactively diagnoses issues and recommends solutions without requiring any human intervention. The proposed concept of 3C Systems would potentially contribute towards $6 \mathrm{G}$ standardization. The automation and orchestration aspects of this research have variety of applications stretched across city infrastructures, retail, business, tourism, health, law, education and travel. A thorough insight to a broad view of $6 \mathrm{G}$ vision has been presented towards envisioned $3 \mathrm{C}$ Systems, while covering its enabling technologies. The experimental results for the proof of concept implementation has been presented. Results affirm the technical capabilities of the concept, to contribute to several industry 5.0 applications including, but not limited to holographic communication, self-driving vehicles, context-aware infrastructure and personalized interfaces.
\end{abstract}

Index Terms-6G, IBN, SON, Industry 5.0, Collaborative Cognitive Communication Systems, Internet of Senses, Computational Psychology, Edge AI, Super Intelligent IoT.

\section{INTRODUCTION}

The next phase of industrial revolution, Industry 5.0 goes beyond automation for goods and services. It put well-being of society at the centre of automation process to ensure rebots and human work collaboratively using cognitive computing capabilities with human touch. Besides variety of $5 \mathrm{G}$ and beyond enabling innovations for application in industry 5.0, new challenges have been observed on the roadmap to $6 \mathrm{G}$. These challenges emphasize the need to conceive social and collaborative human psychology as part of system design. Lack of which may pose threats to sustainable communities and vibrant societies, compelled to opt such technological advancements (while coping with its possible implications to humanity). It calls for establishment of an emerging researchdomain that considers social behaviors/human psychology while developing collaborative cognitive communication systems. The latest advancements in exploiting newer theories and techniques of computational psychology have shown potential to achieve the specified milestones. In order to implement such frameworks, there is need for a systematic understanding of cultural values, norms to investigate behavior patterns and their systemic-linking to Artificial Intelligence (AI), Natural Language Processing (NLP) and communication networks. It would assist in achieving the dream of "Internet of Senses" to deliver multi-sensory

\footnotetext{
${ }^{1}$ This version of paper was accepted on 03 March 2021 for Publication to IEEE Communications Standards Magazine

${ }^{2}$ Corresponding author: Dr. Muddesar Iqbal, email: m.iqbal@1sbu.ac.uk
}

experiences while substituting human intelligence in 6G communication systems. The corresponding author coined the expression "3C Systems" to refer such artifacts at the Cognitive Systems Research Centre(CSRC), London South Bank University. The term stands for Collaborative Cognitive Communication Systems and in the remainder of this article, we intend its use to refer such systems that aim to design and develop self-organized, self-managed, self-monitoring and self-healing applications that relies on human-centric approach to support connected and autonomous intelligent environments for industry 5.0 applications.

$6 \mathrm{G}$ networks are envisaged to support the requisite resources for the fast-paced digital transformation through industry 5.0 application and services. Researchers have already anticipated the potential $6 \mathrm{G}$ enabling technologies to support 3C systems for numerous applications. These applications include, though not limited to, connected robotics, AI assistants, holographic communication, autonomous vehicles, context-aware infrastructure, personalized interfaces, augmented reality and predictive recommendation systems. In asserted range of applications, collaborative cognition will enable 3C systems to mimic human behaviors while providing communication infrastructures to support innovations of industry 5.0. However, it may call for revisiting the long-term technology plans beyond enablement of $5 \mathrm{G}$ and $6 \mathrm{G}$. These enabling technologies will cater the smart cities of future and make socio-economic impact. The designed frameworks for envisioned 3C Systems must enable the use of collected wisdom effectively to cater the needs of individual and group users. It would be possible only by instilling-in cognitive ability to 
understand human's personal and social needs. Ubiquity of these envisioned applications will further enhance unlimited access to contextual information on anything, at anytime, anywhere. With advancements in communication technologies, the dream to travel time and space with human senses in communication is becoming reality through holographic telepresence and augmented projection interfaces. To fulfill these dreams, It requires further research on discussed $6 \mathrm{G}$ enabling technologies to achieve zero-latency milestone by 2030 to reach its full potential [1]. The debate on $5 \mathrm{G}$ and beyond has already kicked off and the first vision of $6 \mathrm{G}$, set by 6 Genesis in Finland is expected in 2030 [2]. A high-level view of core components of $6 \mathrm{G}$ roadmap is represented in Figure 1 .

In this paper, we will focus on to draw a road-map for $6 \mathrm{G}$ enabling technologies to support 3C Systems. A communication framework has been proposed that exploits intelligent Intentbased networking (IBN) infrastructures. It also encompasses open-cloud based APIs to revolutionize the orchestration, resource provisioning, policies and response to user and system events. The proposed IBNs enabled 3C systems may exploit AI, ML and NLP algorithms with psychometric data analytic capabilities. Such autonomous system is able to monitor Content Delivery Network (CDN) and Virtual Network function (VNF) applications. It may analyze and predict the functional as well as non-functional CDN/VNF requirements through digital conversational interfaces also known as AI bots. Currently commercial implementations of AI bots cannot function in collaborative team environments like human. The collaborative and cognitive capabilities of 3C systems will empower AI bots with human cognition to work in more complex environments to function effectively as team player. We have also presented the proof of concept implementation for IBNs enabled 3C systems, which demonstrate the technical capabilities of the concept, with the potential to produce TRL9 ready application.

The remainder of this paper is organized as follows: In section III. The 3C Systems enabling technologies have been elaborated in context of $6 \mathrm{G}$. Section III presents the use case opportunities that may become the key enablers for future $3 \mathrm{C}$ services progression with associated challenges and issues. In section IV, we have propose an Architecture for 3C Systems as proof of concept. Section $\mathrm{V}$ presents the performance evaluation for the proof of concept . Finally, Section VI draws a conclusion and highlights potential future directions.

\section{6G ENABLING TECHNOLOGIES FOR 3C Systems}

In this section, we present the enabling technologies that can be combined to achieve full potential of the envisioned Collaborative Cognitive Communication Systems capabilities.

\section{A. Edge AI Analytics for $6 G$ Networks}

AI is playing a pivotal role in making smarter machines, replicating human behaviors and intelligence to provide cognitive-communication based networks for addressing diverse requirements [3]. To enable the AI on edge, cooperative data intelligence and advanced IoT models play a vital role in fulfilling the needs of novel $6 \mathrm{G}$ applications [4], [5]. AI can be further divided into two levels: First is the deterministic level where multiple pre-defined options are available to respond the environment; second level to bring human cognition into communication by providing the full capability of sensing, mining and prediction from surrounding environment [6]. To enhance the on-demand service provisioning feature, the cellular networks are required to adapt intelligent infrastructure for self-configuration, self- optimization and self-healing applications [6]. Self-finding pattern service can be beneficial to adapt to new trends and be helpful to infer suitable provisioning mechanisms for ever-changing requirements. Moreover, AI analytics play a vital role in implementing given requirements [7]. In this section, we will discuss how AI can play a role in a $6 \mathrm{G}$ cellular networks with smart AI operations and contextaware environments.

1) Smart AI Operations with Digitalization In the last few years, AI market has been growing consistently. AI solutions have enabled a wider variety of applications including smart cities, smart personal assistants, autonomous vehicles, smart metering and smart surveillance to mention a few [4]. Such applications require smart operations with distributed and on-the-fly AI analytics, where AI models can be trained on the go to learn from past data patterns to predict next behavior. Machine learning is the key technology to drive innovation in such interactions by improving efficiency and complexity for disruptive 6G application scenarios [8]. Deep Neural Networks (DNN) are also playing a vital role in wireless communication to provide smart services for end-users with edge intelligence [4]. A few service delivery usecases for 3C systems are presented in Figure 2. The NLP and machine learning is the key technology to enable onthe-fly training capabilities based on contextual learning and psychometric analysis to provide recommendations for these uses cases.

2) Situational and Social Context-Aware Environment The concept of Context-aware environment has been widely used for communication systems to increases situational contents awareness. However, in 3C systems, we propose to use Situational and Social Context-Aware Environments using the process as presented in Figure 2 b. The effectiveness of the envisioned 3C Systems heavily relies on the context in which the information is collected and the method used to process actions. It requires adaptation of context aware collaborative filtering and prediction algorithms to recommend actions based on the social and situational contextual learning.

\section{B. Super Intelligent IoT}

The estimated number of IoT devices have reached between 25-50 billions. With the rapid growth of these hardware and software technologies, massive data is observed to predict and recommend actions for $6 \mathrm{G}$ applications such as self-driving vehicles, autonomous mobility and on-demand connectivity [9]. In Future, Super Intelligent IoT will replace traditional IoT to act as a vital component of $6 \mathrm{G}$ intelligent edge development [4]. 

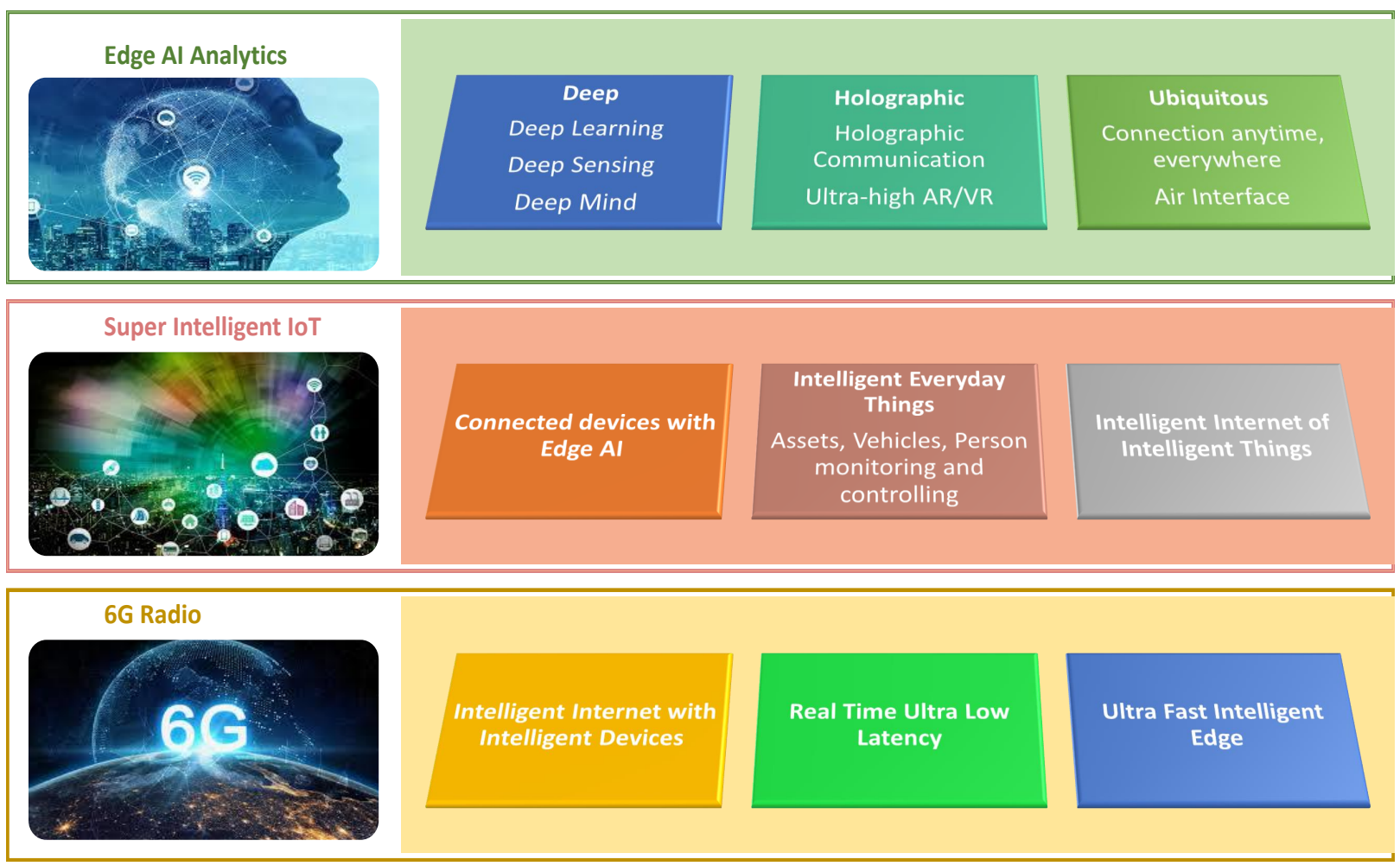

Fig. 1. High Level View of Core Components of $6 \mathrm{G}$

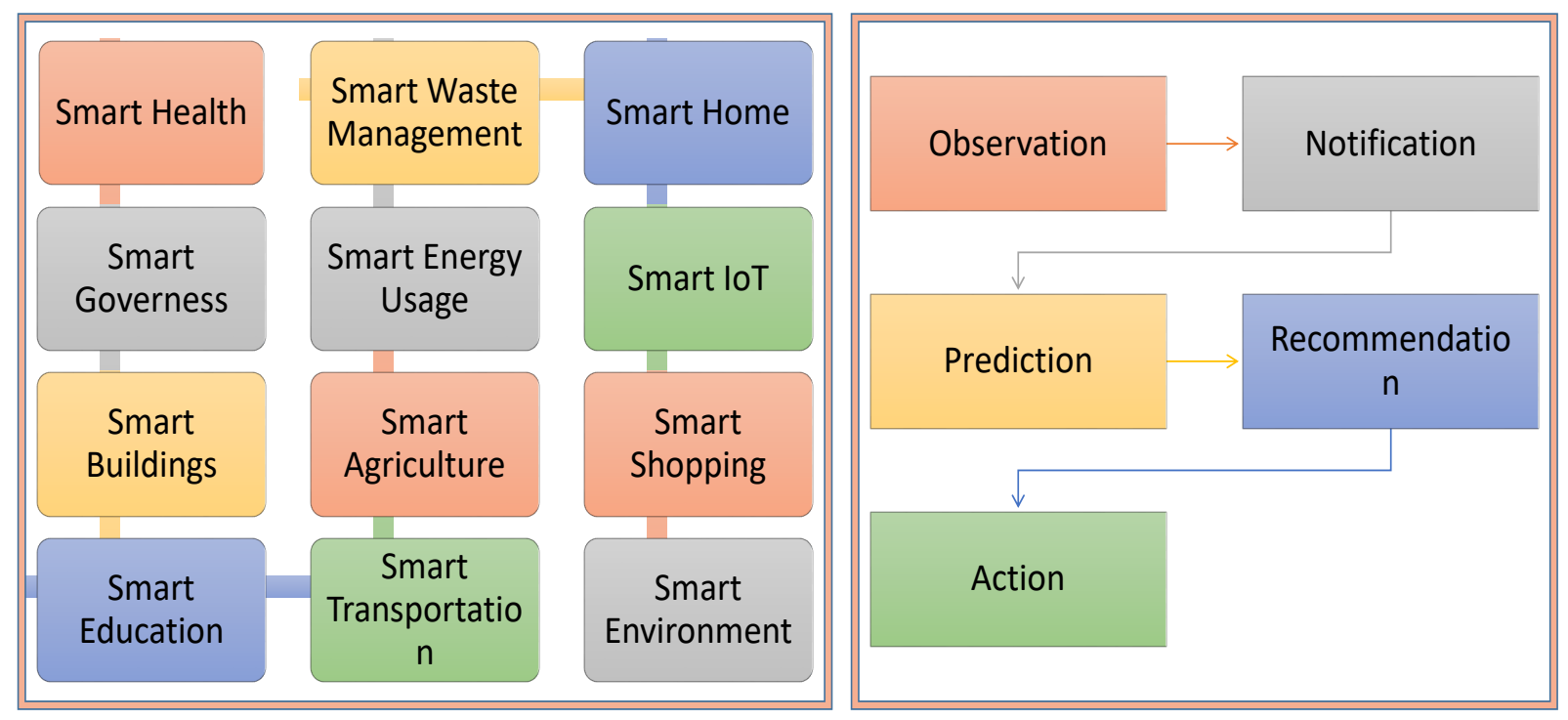

Fig. 2. a) $6 \mathrm{G}$ enabled Application Scenarios b) Role of 3C Systems in $6 \mathrm{G}$ enabled Application Design 
1) Connected Devices with Edge AI .Multi-access edge computing [10] provides a fast solution by reducing the latency rate for smart device communication and gaining contextual information for $6 \mathrm{G}$ enablement [11]. Smart devices are used to gather contextual information that is forwarded to fog layer for data processing. The fog layer offloads this data to edge for analytical processing and forwards it to the cloud data center for model training. The communication between these layers is two-way. The cloud performs the model training on the huge dataset collected from million devices for predictive purpose. This information is passed through the edge layer for seamless operations and later forwards it to the fog for predictive analysis. Fog forwards the information in terms of recommendation or prediction to the end-user devices. The Figure 3 shows 6G Super Intelligent IoT Requirements with Multi Access Edge Computing. AI model training is performed on the cloud with intelligence analytics [4]. The edge node will perform inference and edge analytics on the AI model for seamless operations. Predictive analysis can be performed on the fog node by offloading the data and smart devices for predictive information.

2) Internet of Intelligent Things The extraction of information is going to be a major challenge in IoT application scenarios with billions of devices connected. However, such information extraction methods are key for intelligent $3 \mathrm{C}$ applications. $6 \mathrm{G}$ must enable innovative way to extract live information dynamically to respond in fragile environments while adapting to smart application behaviours. It will open a new dimension of technology by providing intelligent Internet and connected IoT intelligence [1]. The future IoT operations have been presented in Figure 3 Use cases related to 6G massive MTC are discussed in [9]: such as connected industries, swarm networking, personalized body area network, zero energy IoT, Internet of senses and distributed ledger technology. The state-space models for AI techniques play a major role in supporting $3 \mathrm{C}$ systems requiring object recognition, location prediction, sensor fusion, target tracking, pattern discovery, environment mapping, feature extraction, cooperative localization and autonomous navigation.

3) 6G Radio with Multi Access Edge Computing 6G will rely on ultra-fast intelligent edge to fulfill ultra-low latency requirements for future applications [8]. It presents an edge- based architecture for the cellular network and the way machine learning algorithms predict application usage and reduce its response time [4-5]. New services and technologies enablers are emerging to support future smart world application scenarios. Use cases for $6 \mathrm{G}$ radio broadband connectivity are presented in [8] such as: extreme capacity xHaul (backhaul or xHaul), enhanced hotspot, short-range device-to-device communications, smart rail mobility, multi-sensory extended reality, industrial automation and robotics, autonomous mobility and connectivity in remote areas.

\section{3C Systems OpPoRTUNITIES AND CHALLENGES}

In 2040, trillions of IoT connected objects are expected to connect with $5 \mathrm{G}$ and beyond with a variety of smart products and smart services in health, agriculture, shopping, transportation, logistics and sustainable energy. The emergence of analytical intelligence in service provisioning, mobility management and radio resource management has become an important aspect of $6 \mathrm{G}$ cellular networks. Future 6G driving applications are presented in [12] with enabling technologies and driving trends. Application areas for $6 \mathrm{G}$ technology are highlighted in Figure 4

1) Intelligent Personal Edge Intelligent Personal Edge is one of the core requirements for the future networks. These are personalized intelligent interfaces demanded for future applications. For example, personal health analytics or health indicator services sharing via augmented projection interfaces. This personalized AI interfaces will be possible with cross service architecture, wireless multi access connectivity, machine learning, edge analytics and Blockchain.

2) Sensor to AI Fusion Sensor to AI Fusion requires ambient sensing intelligence. For instance, from the autonomous health system, each individual health details i.e. blood sample details are available to scan the health indicator. Smart clothing and environment can also be achieved through sensor to AI fusion. To achieve all the details intelligently from the environment, machine learning, cyber security, edge analytics and Blockchain are required to make it possible.

3) Super Functional Products To enable super functional products in future that may consist of multidimensional design technologies, functional materials, electronics and edge analytics. These super functional product requirements are achievable via dynamic configuration, product gamification, Internet of Things and Hologram User Interfaces.

4) Smart Materials Smart materials required for a smart lifestyle with printed electronic products. For example, electronic printed bottle of a water with all the hydration level details available via personalization, customize UIs and sensors. To achieve this requirement, we need to design 3D IoT, augmented sensing, reusable materials and printed electronics.

5) Mobility as a Service To deal with the user and object mobility, mobility as a service is needed for objects and infrastructure communication with stream analytics. In order to monitor the mobility of moving vehicles for autonomous safety management and logistics guidance, we require the wireless multi-radio connectivity, stream analytics, multi-object IoT and edge computing.

6) Personalized Surfaces Personalized surfaces are required for future applications with printed electronics fused with IoT. These personalized interfaces consist of contextual applications that are interlinked with AI and wireless services. Context aware communication for activity building and context aware content production based on user preferences. This can be achieved via con- 

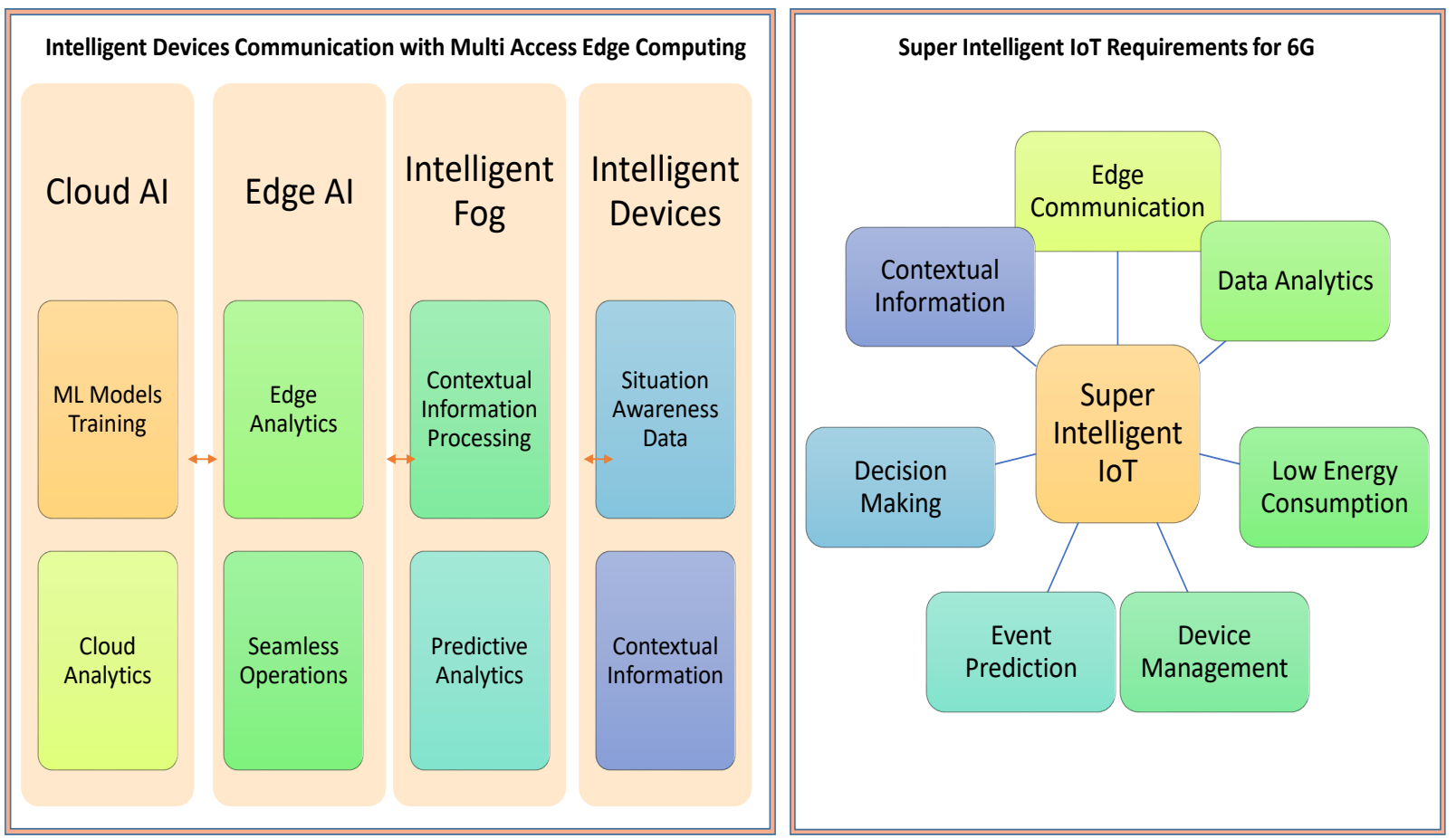

Fig. 3. 6G Super Intelligent IoT Requirements with Multi Access Edge Computing

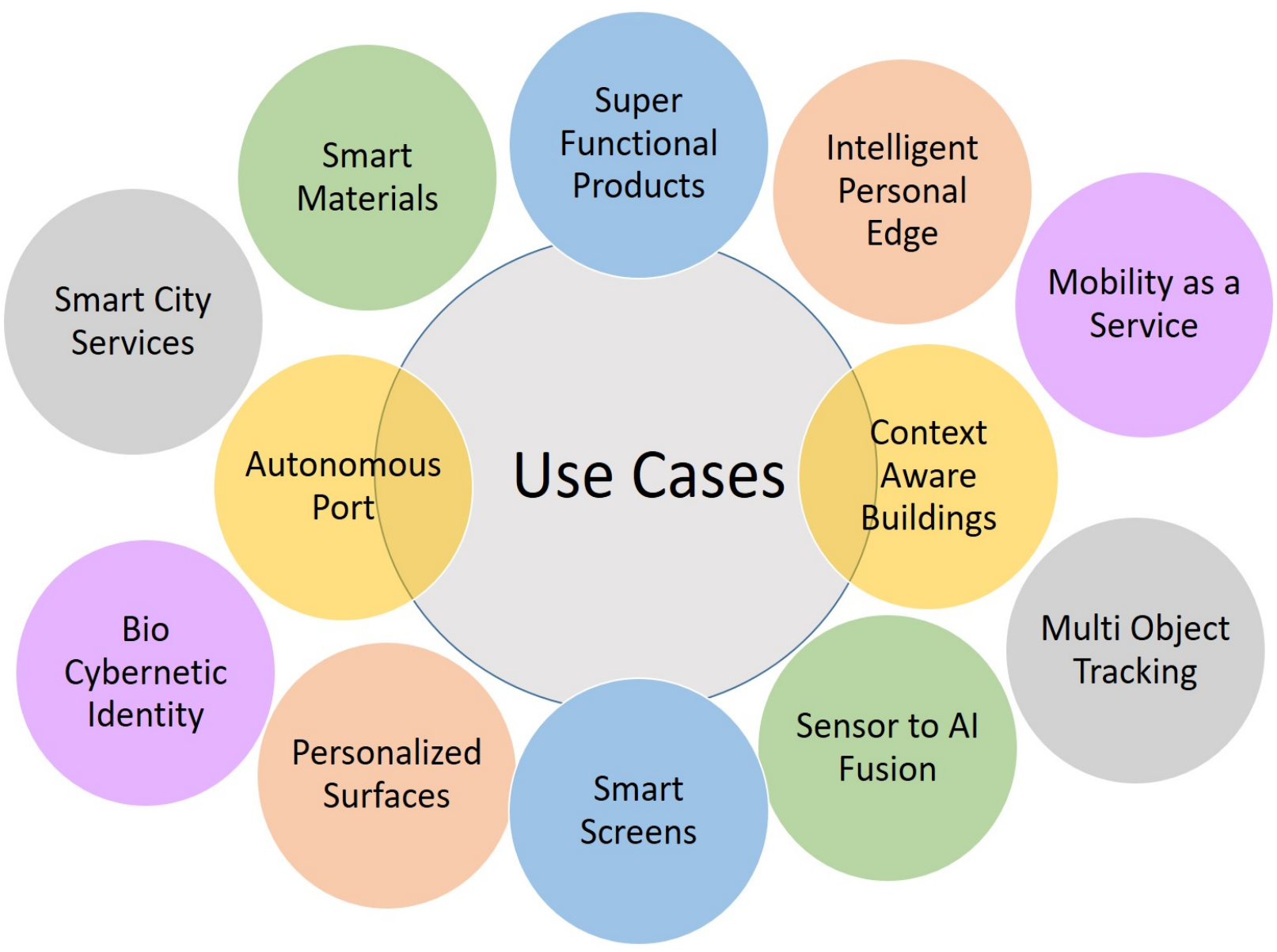

Fig. 4. 6G Use Cases for Industry 5.0 Applications 
sent management, context processing, edge computing and smart surface.

7) Context-Aware Buildings Context Aware building for smart living are required to enable the communication between elements and activity. For instance, automatically light scheduling on the basis of user activity detection. Active building elements are connected with human UI through AI interfaces. Infrastructure IoT, ubiquitous sensing, distributed identity and embedded intelligence are required to achieve this.

8) Multi-Object Tracking To enable multi object tracking, programmed IoT associations, edge connectivity and sensing networks are required to verify the sender identity from the ledger details. All multi-object tracking data will be stored on autonomous system and sender identification will be verified via consent management, swarm analytics, cyber security and blockchain.

9) Bio Cybernetic Identity In order to enable bio cybernetic identity, identical critical service architectures, sensing based machine learning and distribution of trust are required. For example, biometric face scanning feature may verify the identity of sender, context processing, machine learning, cyber identity and biometrics details.

10) Autonomous Port Autonomous port for logistics of merchandise will provide a more transparent way during import and export of products. Swarm based operations and collaborative mobility is highly required to achieve this by using wireless Internet of Things, system interoperability, data patterns analytics and stream analytics.

11) Smart Screens Smart Screens for smart applications need programmable augmented materials. In order to develop the habit tracking system, context aware content production and preference based user experience are highly in demand. This can be achieved using multichannel connectivity, printed electronics, context aware IoT and smart surface

In above-mentioned use cases, 5G has a lack of support and limitations to fulfill these requirements. However, 6G also has some potential issues and obstacles. [1], [13] [14]. These challenges may threaten the well-being and livelihood of our societies, with several implications such as economic, social, political, cultural, environmental and technical ones. Therefore, 3C systems must address these challenges as part of the application design, by developing better understanding on these socio-economic challenges.

\section{PROOF OF CONCEPT}

In Figure 5,we have proposed an innovative framework for Collaborative Cognitive Communication (3C) systems and implemented a Proof of Concept to build the technical and theoretical awareness. The implementation exploits the technical capabilities of the Intent based Networking (IBN) concept to develop infrastructure framework with emerging open-based cloud APIs and AI assistant. The use-case focuses on providing end-to-end automation for cloud orchestration, resource provisioning and policies. It has implementation based on the AI virtual assistant that learns on user's contextual information and business needs. In order to achieve this, proposed framework exploits AI, ML and NLP algorithms with Psychometric data analytic capabilities to develop autonomous Content Delivery Network (CDN) and Virtual Network function (VNF) deployment. In proposed architecture, the 3C systems analyze and predict functional as well as non-functional CDN/VNF requirements while communicating to the non-technical users through digital conversational assistant interfaces. These interfaces have been developed and integrated using RASA open source libraries. We have programmed dynamic APIs, those make use of NLP and ML, LibCloud APIs to empower the Virtual Assistant (Chabot) to function in collaborative manner like human. The chatbot interface is capable of performing the cognitive tasks to function effectively as team player. The Figure 5 illustrate the proof of concept architecture implementation while integrating several technologies and their flow as captioned in Figure 5 .

\section{Proof of Concept Performance Evaluation}

In order to understand the proof of concept implementation for the proposed 3C framework, a situation has been narrated as follows: Let's suppose, John is a big fan of football. He organizes live football matches from pubs. Due to Covid19 social distancing restrictions in place for pub gatherings, John sets up a website for his business. He needs to deploy a cloud based VNF/CDN for Over-the-top (OTT) streaming [15] through his website for a popular match in multiple regions. John $\log$ s on the cloud provider and chats away his requirements using non-technical requirements with the chatbot based AI assistant. The AI assistant uses NLP algorithms to convert the non-technical description into the technical functional requirements. These requirements are passed onto the cloud orchestration API that is designed and implemented to perform both centralized resource management and distributed VNF placements. It uses on the fly VNF/CDN placement mechanism for OTT streaming that works on orchestrator level. The orchestrator initiates the deployment of VNFs for Jonh's OTT using multiple streaming and transcoding containers. John has also specified a minimum QoE requirement that must be maintained at all times during the match broadcast. The designed hypervisor uses its ability to assign additional resources (i.e. virtual CPUs, RAMs) to instantiate additional VMs or expand the existing resources available for existing VMs to maintain the required minimum QoE level. We have developed the orchestrator as a resource controller using LibCloud libraries which responds to the live events and triggers actions to adjust OpenStack cloud resources to meet the QoE needs e.g. in case, the number of viewers exceed the limit communicated by John during his initial conversation with the AI assistant, orchestrator would take actions such as deploying more VMs/Containers or migrating the VMs/Containers from under-utilized regions to the region with higher traffic load for maintaining the required quality of experience during match streaming. It is also capable of managing event of failure 


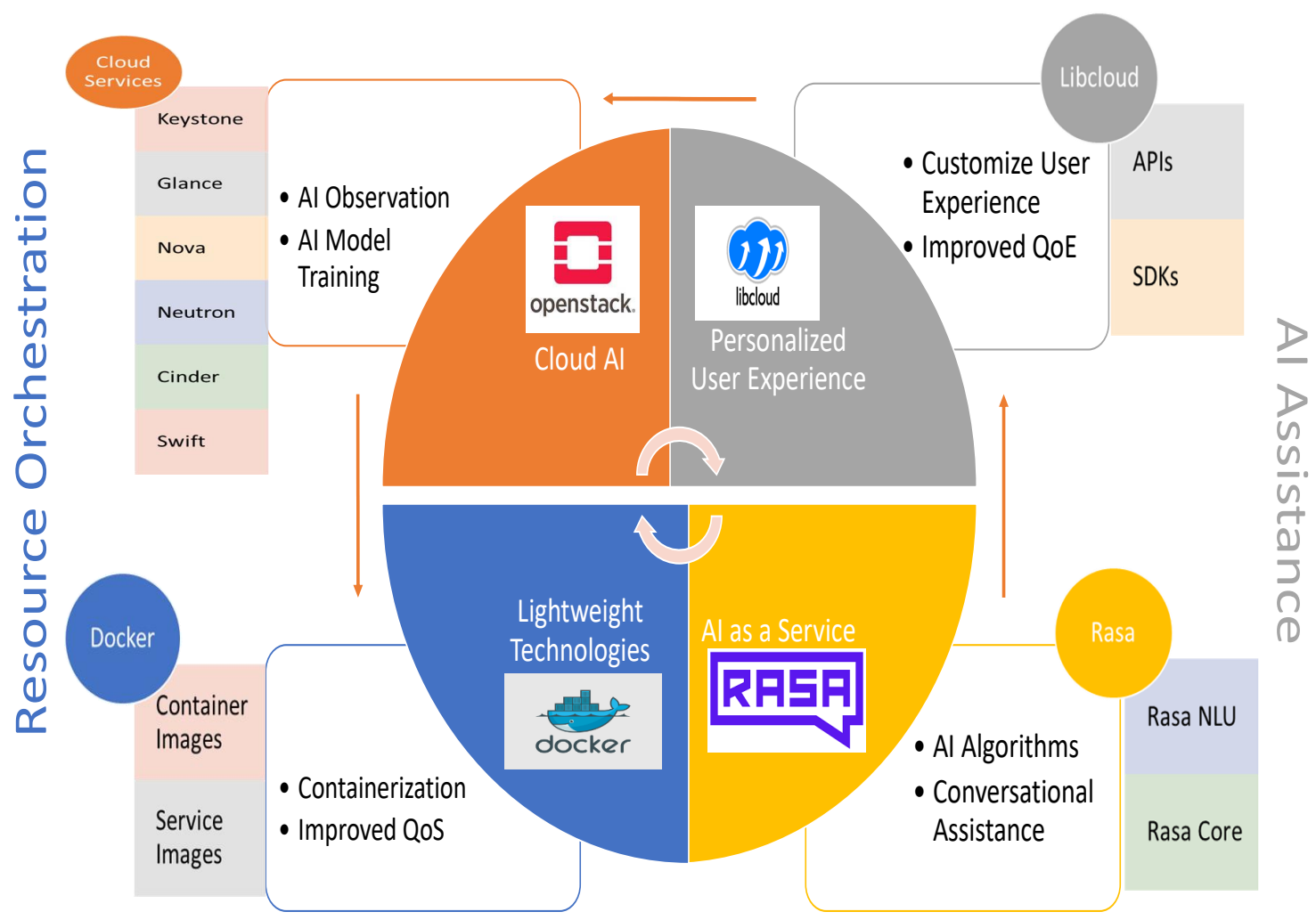

Fig. 5. Proof of Concept Framework Implementation Architecture for 3C Systems Use-case Scenario

on the fly by balancing the CPU power consumption and assignment to stabilize hardware performance and potentially save power. The cloud orchestration API module alerts the orchestrator when resources become available, allowing the orchestration system to use live migration to swap around the workspaces for optimizing the overall resources. The orchestrator calculates the cost of the QoE using the centralized approaches to ensure that the resources exist in groups that accurately match the expected resource consumption of each expected workspace. When the cloud orchestration API receives data related to types of flows being requested with the current status of the resources. The cloud API orchestrator dynamically diverges from the centralized resource management model to distributed model. It allocates resources individually by drawing the resources from a pool of compute nodes that are hosted in geographically different countries/regions. While adopting the distributed model, the cloud API orchestrator has the ability to perform live migration of allocated resources and terminate the resources. The orchestrator provides the resource allocation based on the load requirements of the users. Using the proposed mechanism in this paper, the cloud orchestrator API is forced to wait and allow the high cost flows to complete. It potentially avoids the termination and instantiating or reallocating resource costs that would normally be associated with collecting and returning resources from a resource pool allocation costs source. The Cloud Orchestration API also makes use of health indicators that we define to represent the current state of the CPU's. It passes along the information to the orchestrator. It will then make the decision to either reallocate the flows to a new CPU or to give back the $\mathrm{CPU}$ to the pool if the flow requests do not require enough resources. Each decision, the Cloud Orchestration API takes , tries to achieve better performance under high load and exchanges deteriorate performance (due to CPU reallocation) with a better Quality of Experience (QoE). The detailed description of experimental results are explained in following sections. The scope of performance analysis as shown in Figure 6 is focused on the cloud performance.

\section{A. Delay Calculation}

During the VNF/CDN deployment, We have used a $1 \mathrm{MB}$ size file name as "test $1 \mathrm{Mb}$.db" to measure the downloading delay from central cloud and local edge servers. This would give us an idea how much delay we should expect when the orchestrator fulfill the requested call.

- Downloading delay from central server (i.e. controller server): $21.08 \mathrm{sec}$

- Downloading delay from local server 1 (i.e. object1 server): $11.45 \mathrm{sec}$ 


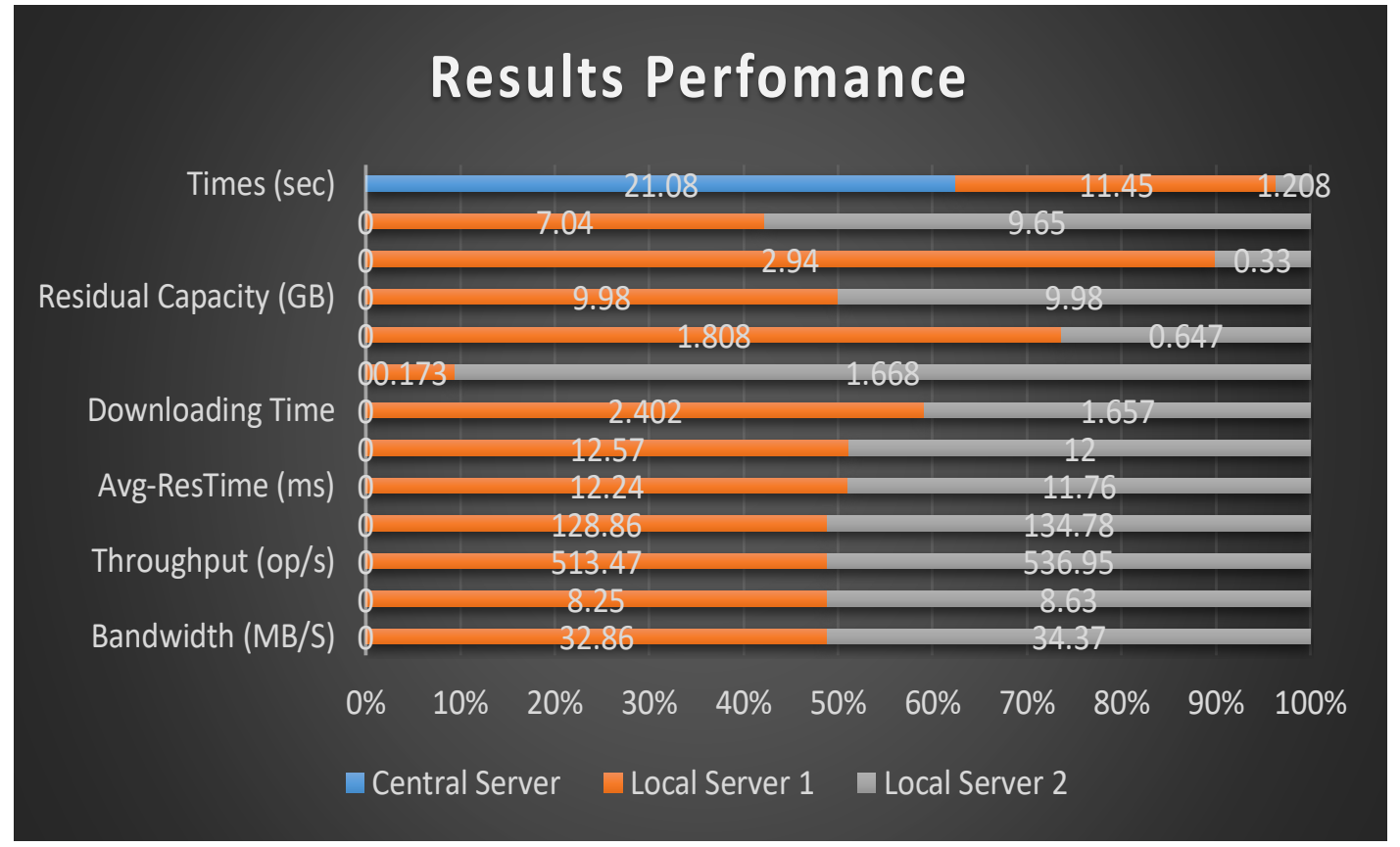

Fig. 6. Results Performance

- Downloading delay from local server 2 (i.e. object2 server): $1.208 \mathrm{sec}$

\section{B. Bandwidth Calculation}

To measure the bandwidth of local edge servers, we have used $1 \mathrm{MB}$ object/file size for demo.

Bandwidth from Local Edge Server 1:

- For read operation: $32.86 \mathrm{MB} / \mathrm{S}$

- For write operation: $8.25 \mathrm{MB} / \mathrm{S}$

Bandwidth from Local Edge Server 2:

- For read operation: $34.37 \mathrm{MB} / \mathrm{S}$

- For write operation: $8.63 \mathrm{MB} / \mathrm{S}$

\section{Throughput Calculation}

The power management controller as an interface between the deployed orchestrator. The power controller communicates to the cloud orchestration API when resources become available. Using the framework illustrated in Figure 5, the orchestrator triggers the live migration to swap around the workspaces to optimize the overall resources. We have implemented the dynamic migration of VMs to adjust the resources allocated to a workspace based on their resources requirements and resource consumption of the workspace. The throughput available will affect this dynamic migration, therefore it is important to understand how much it cost to perform these read and write operations dynamically. In order to understand this, we have measured the throughput of local Edge servers, we have used $1 \mathrm{MB}$ object/file size for demo.

Throughput from Local Edge Server 1:

- For read operation: $513.47 \mathrm{op} / \mathrm{s}$

- For write operation: $128.86 \mathrm{op} / \mathrm{s}$

Throughput from Local Edge Server 2:

- For read operation: $536.95 \mathrm{op} / \mathrm{s}$

- For write operation: $134.78 \mathrm{op} / \mathrm{s}$

\section{Response Time}

In the proof of concept implementation, we have allocated resources for individual user which contains a unique connection to the cloud orchestration API. In order to measure 
the effects, we have calculated the average response time and residual capacity from local edge servers. We used $1 \mathrm{MB}$ object/file size for demo for the average response time.

Average Response Time from Local Edge Server 1:

- For read operation: $12.24 \mathrm{~ms}$

- For write operation: $12.57 \mathrm{~ms}$

Average Response Time from Local Edge Server 2:

- For read operation: $11.76 \mathrm{~ms}$

- For write operation: $12 \mathrm{~ms}$

\section{E. Residual capacity}

- Residual capacity of Local Edge Server 1:

- Total Capacity: 10716446720 bytes $=9.98$ GB

- Used Capacity: 3161657344 bytes $=2.94 \mathrm{~GB}$

- Residual Capacity: 7554789376 bytes $=7.04$ GB

- Residual capacity of Local Edge Server 2:

- Total Capacity: 10716446720 bytes $=9.98$ GB

- Used Capacity: 354062336 bytes $=0.33 \mathrm{~GB}$

- Residual Capacity: 10362384384 bytes $=9.65$ GB

\section{F. Load Performance Measurement}

The load performance of the local edge servers will effect how the model performs, therefore in order to understand the effects, we have presented the Load Performance of local edge server 1 and 2 and here are the outcomes. Load Performance of Local Edge Server 1: When 3 users simultaneously try to download the object of $1 \mathrm{~KB}$ size. Downloading Time: $2.402 \mathrm{sec}$ When 3 users simultaneously try to download the object of $2 \mathrm{~KB}$ size. Downloading Time: $0.173 \mathrm{sec}$ When 3 users simultaneously try to download the object of $5 \mathrm{~KB}$ size. Downloading Time: $1.808 \mathrm{sec}$

Load Performance of Local Edge Server 2: When 3 users simultaneously try to download the object of $1 \mathrm{~KB}$ (1024) size. Downloading Time: $1.657 \mathrm{sec}$ When 3 users simultaneously try to download the object of $2 \mathrm{~KB}$ (2048) size. Downloading Time: $1.668 \mathrm{sec}$ When 3 users simultaneously try to download the object of $5 \mathrm{~KB}$ (5120) size. Downloading Time: $0.647 \mathrm{sec}$

The delay bandwidth and throughput experimental results presented in section $\mathrm{A}, \mathrm{B}$ and $\mathrm{C}$ demonstrates the technical capabilities of all the integrated technologies to a Technology readiness Level (TRL) 3. The Proof-of-Concept demonstrate the feasibility and verifying that the proposed concept has practical potential to further developed a TRL 9 Collaborative Cognitive Communication (3C) System, to support industry 5.0 application areas for $6 \mathrm{G}$ enabled self-organised, selfmanaged, self-monitoring and self-healing application technologies.

\section{Vi. Conclusion and Future Directions}

In this article, we have proposed our $6 \mathrm{G}$ vision towards Collaborative Cognitive Communication (3C) Systems. The paper provides an understanding to some key enabling technologies vital to set $6 \mathrm{G}$ roadmap for $3 \mathrm{C}$ systems. We have also discussed several opportunities that may become the key enablers for future $3 \mathrm{C}$ service developments. It is also highlighted to address the corresponding socio-economic challenges and issues likely to impact human lives. An innovative architecture for 3C Systems has been proposed to demonstrates the technical capabilities as proof of concept. Although, the proof of concept focuses on the CDN/NFV, the same concept is applicable to impact every sector of the economy including infrastructures, retail, business, tourism, health, law, education and travel. The performance evaluation of on-the-fly cloud $\mathrm{VNF} / \mathrm{CDN}$ deployment is presented to demonstrate the feasibility and capabilities of all the integrated technologies to work as single $3 \mathrm{C}$ system to a Technology Readiness Level (TRL) 3. The performance evaluation results verify that the concept has the potential to produce a fully functional TRL 9 Collaborative Cognitive Communication (3C) Systems for industry 5.0 application.

In future, we plan to publish the theoretical details for the implemented framework and associated algorithms that enabled the implementation of Collaborative Cognitive Communication within the proposed architecture.

\section{REFERENCES}

[1] M. Matinmikko-Blue, S. Aalto, M. I. Asghar, H. Berndt, Y. Chen, S. Dixit, R. Jurva, P. Karppinen, M. Kekkonen, M. Kinnula et al., "White paper on $6 \mathrm{~g}$ drivers and the un sdgs," arXiv preprint arXiv:2004.14695, 2020.

[2] M. Katz, P. Pirinen, and H. Posti, "Towards 6g: Getting ready for the next decade," in 2019 16th International Symposium on Wireless Communication Systems (ISWCS). IEEE, 2019, pp. 714-718.

[3] X. Wang, X. Li, and V. C. Leung, "Artificial intelligence-based techniques for emerging heterogeneous network: State of the arts, opportunities, and challenges," IEEE Access, vol. 3, pp. 1379-1391, 2015.

[4] E. Peltonen, M. Bennis, M. Capobianco, M. Debbah, A. Ding, F. GilCastiñeira, M. Jurmu, T. Karvonen, M. Kelanti, A. Kliks et al., "6g white paper on edge intelligence," arXiv preprint arXiv:2004.14850, 2020.

[5] L. Lovén, T. Leppänen, E. Peltonen, J. Partala, E. Harjula, P. Porambage, M. Ylianttila, and J. Riekki, "Edgeai: A vision for distributed, edgenative artificial intelligence in future $6 \mathrm{~g}$ networks," The 1st $6 \mathrm{G}$ Wireless Summit, pp. 1-2, 2019.

[6] R. Li, Z. Zhao, X. Zhou, G. Ding, Y. Chen, Z. Wang, and H. Zhang, "Intelligent $5 \mathrm{~g}$ : When cellular networks meet artificial intelligence," IEEE Wireless communications, vol. 24, no. 5, pp. 175-183, 2017.

[7] M. S. Mahdavinejad, M. Rezvan, M. Barekatain, P. Adibi, P. Barnaghi, and A. P. Sheth, "Machine learning for internet of things data analysis: A survey," Digital Communications and Networks, vol. 4, no. 3, pp. 161-175, 2018.

[8] N. Rajatheva, I. Atzeni, E. Bjornson, A. Bourdoux, S. Buzzi, J.-B. Dore, S. Erkucuk, M. Fuentes, K. Guan, Y. Hu et al., "White paper on broadband connectivity in 6g," arXiv preprint arXiv:2004.14247, 2020.

[9] N. H. Mahmood, S. Böcker, A. Munari, F. Clazzer, I. Moerman, K. Mikhaylov, O. Lopez, O.-S. Park, E. Mercier, H. Bartz et al., "White paper on critical and massive machine type communication towards $6 \mathrm{~g}$," arXiv preprint arXiv:2004.14146, 2020.

[10] T. D. a. Z. U. Q. Sonia Shahzadi, Muddesar Iqbal, "Multi-access edge computing: open issues, challenges and future perspectives," Journal of Cloud Computing, vol. 30, no. 6, pp. 1-13, 2015.

[11] N. H. Mahmood, H. Alves, O. A. López, M. Shehab, D. P. M. Osorio, and M. Latva-aho, "Six key enablers for machine type communication in 6g," arXiv preprint arXiv:1903.05406, 2019.

[12] W. Saad, M. Bennis, and M. Chen, "A vision of $6 \mathrm{~g}$ wireless systems: Applications, trends, technologies, and open research problems," IEEE network, vol. 34, no. 3, pp. 134-142, 2019.

[13] M. H. Alsharif, A. H. Kelechi, M. A. Albreem, S. A. Chaudhry, M. S. Zia, and S. Kim, "Sixth generation (6g) wireless networks: Vision, research activities, challenges and potential solutions," Symmetry, vol. 12, no. 4 , p. $676,2020$.

[14] H. Saarnisaari, S. Dixit, M.-S. Alouini, A. Chaoub, M. Giordani, A. Kliks, M. Matinmikko-Blue, N. Zhang, A. Agrawal, M. Andersson et al., "A $6 \mathrm{~g}$ white paper on connectivity for remote areas," arXiv preprint arXiv:2004.14699, 2020. 
[15] U. Bulkan, M. Iqbal, and T. Dagiuklas, "Load-balancing for edge qoebased vnf placement for ott video streaming," pp. 1-6, 2018. 